\title{
A Comparison of the Sociodemographic and Clinical Characteristics of Patients Referring to a Pain Clinic with Subacute and Chronic Pain
}

\author{
Seyed Masoud Hashemi, ${ }^{1}$ Ramin Rohanifar, ${ }^{2}$ Rasoul Azarfarin, ${ }^{3}$ Seyed Sajjad Razavi, ${ }^{1}$ and Sirous
}

\author{
Momenzadeh ${ }^{4, *}$ \\ ${ }^{1}$ Department of Anesthesiology, Shahid Beheshti University of Medical Sciences, Tehran, Iran \\ ${ }^{2}$ Department of Anesthesiology, Tehran Clinic Hospital, Tehran, Iran \\ ${ }^{3}$ Rajaie Cardiovascular, Medical and Research Center, Iran University of Medical Sciences, Tehran, Iran \\ ${ }^{4}$ Functional Neurosurgery Research Center, Shahid Beheshti University of Medical Sciences, Tehran, Iran \\ "Corresponding author: Sirous Momenzadeh, Department of Anesthesiology, Anesthesia Research Center and Functional Neurosurgery Research Center, Shohada Tajrish \\ Neurosurgical Center of Excellence, Shahid Beheshti University of Medical Sciences, Tehran, Iran. Tel: +98-2122612252, Fax: +98-2122663293, E-mail: \\ raminrohanifar@yahoo.com
}

Received 2016 May 21; Revised 2016 July 12; Accepted 2016 September 06.

\begin{abstract}
Objectives: The aim of the present study was to assess and compare the sociodemographic characteristics and clinical features of patients referring to a university hospital's pain clinic with chronic ( $\geq 12$ weeks) and subacute pain $(<12$ weeks).

Methods: In this cross-sectional study, 426 patients were included. Demographic variables including education level, marital and employment status, and risk factors such as obesity, diabetes mellitus, hypertension, cigarette smoking, and opium addiction were recorded. Also, sites of pain, pain quality and associated symptoms, and pain severity were assessed using a numerical rating scale. Each one of these variables was compared between the chronic and subacute pain groups.

Results: Of the 426 studied patients, 292 (69\%) had chronic pain and 134 (31\%) reported subacute pain. Patients with chronic pain were older and had higher body mass indices. Additionally, self-employment was less frequent among the chronic pain group. The patients with chronic pain had a higher prevalence of addiction. The most commonly reported site of pain in all patients was the lower back (62.4\%), followed by pain in the leg and foot (39.9\%), knee (24.4\%), and hip (18.8\%). There were no statistically significant differences in pain sites between the two groups, except for knee pain, which was more common among the chronic pain group. The patients with chronic pain had a higher incidence of obscure and persistent pain, while those with subacute pain experienced more night pain.

Conclusions: About one-third of the patients referring to the pain clinic had subacute pain. The patients with chronic pain were older and more obese, had a higher prevalence of addiction, had more cases of knee pain, and reported more instances of obscure and persistent pain than those with subacute pain.
\end{abstract}

Keywords: Chronic Pain, Pain Clinic, Demographic Factors, Epidemiology

\section{Background}

Chronic pain is defined as a sensation of pain which lasts longer than three months (1). The prevalence of chronic pain is common all over the world, and various researchers have estimated that about $7 \%-55 \%$ of the general population ( $20 \%$ on average) have various forms of chronic pain $(2,3)$. Chronic pain may be affected or exacerbated by a variety of environmental, sociodemographic, and individual factors (3-5). Quality of life is considerably affected not only for these patients themselves, but also for their families and friends (4). This type of pain is associated with incomplete treatment and other problems such as sleep abnormalities, chronic fatigue syndrome, drug abuse, loss of appetite, physical disability, anxiety, and depression (6, 7). There is a great deal of evidence showing that chronic pain has an unfavorable influence on physical health, men- tal health, daily activity, employment, and financial wellbeing (2-7).

The epidemiology of chronic pain and its relationship with sociodemographic factors have been reported by numerous studies from various geographical regions and countries, and also among populations with different (low, middle, and high) income statuses across the globe (8). However, relevant data from Middle Eastern countries, including Iran, are limited. Zarei and colleagues (2012) (6) reported a prevalence rate of $38.9 \%$ for chronic pain. They also reported a significant relationship between chronic pain and age, sex, weight, income, educational level, and type of occupation. Those with low incomes and low educational levels coped with pain more effectively owing to a lack of knowledge. In a similar study, Asghari et al. (2008) (7) showed that chronic pain patients with higher education and higher pain self-efficacy beliefs were less physi- 
cally disabled and less depressed.

Some researchers have tried to differentiate between subacute and chronic pain in terms of demographic and clinical features with a view to developing different treatment approaches for each individual patient referring to pain clinics (9-11). Although patients with subacute pain constitute approximately one-third of all patients referring to pain clinics, the existing literature contains a dearth of data on the demographic and clinical features of such patients (11). Moreover, most studies on subacute pain have focused on lower back pain, while most patients referring to pain clinics with subacute pain have various forms of pain in different anatomical sites.

\section{Objectives}

As a result of the limited available data, we sought to evaluate and compare the sociodemographic characteristics and clinical features of patients with chronic ( $\geq 12$ weeks) and subacute pain ( $<12$ weeks) referring to a university hospital's pain clinic in Tehran, Iran.

\section{Methods}

\subsection{Participants}

This cross-sectional study was conducted on 440 patients who reported with the chief complaint of pain to the pain clinic of Shaheed Beheshti University of Medical Sciences, Tehran, Iran, between January and April 2016. Of the 440 patients, nine individuals refused to participate in the study, and five participants had incomplete data. Finally, data on 426 patients were recorded and entered for analysis. Subacute pain is generally defined as pain lasting between four and 12 weeks, and chronic pain is defined as pain that lasts $\geq 12$ weeks (11). The inclusion criteria comprised having a history of subacute or chronic pain, an age $\geq 18$ years old, the ability to speak and read in Farsi, and willingness to participate in the research project. Patients were excluded from the study if they had any type of cancer or overt opium addiction.

\subsection{Measurements}

The demographic variables were comprised of sex, age, residency in Tehran or other cities, education level, marital status, and employment status. Medical history consisted of risk factors such as obesity, diabetes mellitus, hypertension, cigarette smoking, and opium addiction. Data on pain duration, pain site, and average pain intensity as well as information regarding health care and drug usage for pain relief were recorded.
Pain intensity was assessed using a numerical rating scale. On this scale, the patients needed to rate their pain severity on an 11-point scale ranging from 0 to 10 , where 0 showed "no pain" and 10 signified "worst possible pain."

\subsection{Statistical Analysis}

The collected data were analyzed with SPSS statistical software, version 22.0 (SPSS/IBM Inc. Chicago, IL, USA). The participants with chronic pain were compared with those who had pain durations of $<12$ weeks in terms of the continuous variables using a series of independent sample ttests. The assumption of equal variance between the two groups was tested using Levene's test. The categorical variables were compared between the study subgroups using a chi-squared or Fisher's exact test. Statistical significance was considered to be a P value $\leq 0.05$.

\section{Results}

Of the 426 studied patients, 134 (31.5\%) had pain durations of $<12$ weeks (categorized in the subacute group) and 292 (68.5\%) had pain durations $\geq 12$ weeks (categorized in the chronic pain group). All of the demographic and clinical variables were then compared between these two subgroups.

Table 1 demonstrates the demographic parameters of the patients. Most of the patients referring to the pain clinic were women (250/426;58.7\%). As is presented in Table 1 , the patients who referred to the pain clinic with chronic pain were older ( $57.2 \pm 15.3$ vs. $53.5 \pm 15.9 \mathrm{y} ; \mathrm{P}=0.022)$ and had higher body mass indices (BMI) than the patients with subacute pain; moreover, occupational status was different between the two groups, in that self-employment was less frequent among those in the chronic pain group. As is shown in Table 2, among the clinical risk factors, only opium addiction was significantly different between the two groups, and the patients who had longer histories of pain had higher prevalence rates of opium addiction $(5.5 \%$ vs. $1.5 \% ; \mathrm{P}=0.006$ ).

The most commonly reported site of pain among all patients was lower back pain $(n=266 ; 62.4 \%)$, followed by pain in the leg and foot $(\mathrm{n}=170 ; 39.9 \%)$, knee $(\mathrm{n}=104$; $24.4 \%$ ), hip ( $n=80 ; 18.8 \%)$, and neck $(n=44 ; 10.3 \%)$. There were no statistically significant differences in the pain sites between the chronic and subacute pain groups, except for knee pain, which presented more frequently in the chronic group $(\mathrm{n}=88 ; 30.1 \%)$ than in the subacute group $(\mathrm{n}=16$; $11.9 \%)(P=0.001)$. Table 3 summarizes the anatomic sites of pain for both study groups. Table 4 depicts the distribution of the quality of pain and the associated symptoms among the patients in the two study groups. The patients 
Table 1. Demographic Characteristics of the Chronic and Subacute Pain Groups

\begin{tabular}{|c|c|c|c|}
\hline & Chronic Pain, $n=292$ & Subacute Pain, $n=134$ & P Value \\
\hline Sex, $M$ & $178(61.0 \%)$ & $72(53.7 \%)$ & 0.193 \\
\hline Age, $y$ & $57.2 \pm 15.3$ & $53.5 \pm 15.9$ & 0.022 \\
\hline Weight, kg & $74.7 \pm 14.4$ & $71.0 \pm 17.2$ & 0.052 \\
\hline Height, $\mathrm{cm}$ & $164 \pm 13.9$ & $166.9 \pm 11.9$ & 0.187 \\
\hline Body mass index & $27.4 \pm 5.9$ & $25.7 \pm 5.1$ & 0.010 \\
\hline Married & $246(84.2 \%)$ & $102(76.1 \%)$ & 0.182 \\
\hline Education, associate degree and higher & $186(63.7 \%)$ & $88(65.4 \%)$ & 0.846 \\
\hline Job, self-employment & $98(33.6 \%)$ & $58(43.3 \%)$ & 0.041 \\
\hline First referral to a pain clinic & $265(90.9 \%)$ & $105(78.6 \%)$ & 0.336 \\
\hline
\end{tabular}

Table 2. Clinical Risk Factors Among the Chronic and Subacute Pain Groups

\begin{tabular}{|c|c|c|c|}
\hline & Chronic Pain, $n=292$ & Subacute Pain, $n=134$ & P Value \\
\hline Diabetes mellitus & $36(12.3 \%)$ & $12(9.0 \%)$ & 0.391 \\
\hline Hypertension & $52(17.8 \%)$ & $16(11.9 \%)$ & 0.164 \\
\hline Cigarette smoking & $28(9.6 \%)$ & $22(16.4 \%)$ & 0.173 \\
\hline Opium addiction & $16(5.5 \%)$ & $2(1.5 \%)$ & 0.006 \\
\hline History of asthma & 0 & $2(1.5 \%)$ & 0.184 \\
\hline History of rheumatic diseases & $4(1.4 \%)$ & 0 & 0.412 \\
\hline Obesity & $59(20.2 \%)$ & $20(14.9 \%)$ & 0.243 \\
\hline Overweight & $95(32.5 \%)$ & $36(26.8 \%)$ & 0.287 \\
\hline
\end{tabular}

with chronic pain had a higher incidence of obscure (12.3\%) and persistent (19.9\%) pain than those with subacute pain, whereas the latter group experienced night pain more often $(14.9 \%$ vs. $8.2 \% ; \mathrm{P}=0.048)$. There were no statistically significant differences between the associated psychological symptoms, such as sleep disorder, anxiety, and depression, between the two study groups (Table 4). Furthermore, the patients with chronic pain reported higher severity of pain (on the numerical rating scale) than the patients with subacute pain (Table 5).

\section{Discussion}

It is essential to know that the multiple aspects of chronic pain cannot be explained by considering nociception only. It is obvious that genetic, sociodemographic, and clinical factors allied with acute, subacute, and chronic pain are important in recognizing and planning relevant diagnoses and treatment modalities. Current investigations require the integration of epidemiological studies with the clinical assessment and management of acute, subacute, and chronic pain (12).

Chronic pain is a common public health problem in that it affects one-fifth of adults worldwide (13). Pain relief, either medically or surgically, is an essential human right and is a responsibility of physicians especially anesthesiologists working in pain clinics (14-16). Also, about $20 \%$ of Europeans complain of chronic pain (12). However, a considerable portion of the patients who refer to pain clinics have a history of pain $<12$ weeks, and as such cannot be classified as having "chronic pain." In the present study, approximately one-third (31.5\%) of the 426 patients who referred to this particular university pain clinic had a past history of pain $<12$ weeks, categorized as "subacute pain." The epidemiology of chronic pain has been extensively studied in the literature $(17,18)$, but there is little data regarding the sociodemographic and clinical factors of patients referring to pain clinics with subacute pain. The aim of the current study was to evaluate and compare the demographic and clinical features of patients referring to a university hospital's pain clinic with chronic and subacute pain.

As is shown in Table 1, most of the patients who referred to the pain clinic were women (250/426; 58.7\%). Also, chronic pain was relatively more frequent than subacute pain among the female patients (61\% vs. $53.7 \%$ ); however, this difference was not statistically significant. Generally, women tend to exhibit a lower pain sensation threshold and refer to physicians more commonly than men (12). Torrance et al. (2006) (3) reported that $52.3 \%-60.1 \%$ of the patients presenting with chronic pain in different cities of 
Table 3. Anatomic sites of Pain Among the Chronic and Subacute Pain Groups ${ }^{\mathrm{a}}$

\begin{tabular}{|c|c|c|c|}
\hline & Chronic Pain, $n=292$ & Subacute Pain, $n=134$ & P Value \\
\hline Back & $190(65.1 \%)$ & $76(56.7 \%)$ & 0.122 \\
\hline Hip & $56(19.2 \%)$ & $24(17.9 \%)$ & 0.859 \\
\hline Leg & $120(41.1 \%)$ & $50(37.3 \%)$ & 0.526 \\
\hline Knee & $88(30.1 \%)$ & $16(11.9 \%)$ & 0.001 \\
\hline Wrist & $24(8.2 \%)$ & $6(4.5 \%)$ & 0.231 \\
\hline Head & $8(2.7 \%)$ & 0 & 0.121 \\
\hline Face & $2(0.7 \%)$ & $2(1.5 \%)$ & 0.794 \\
\hline Jaw & $6(2.1 \%)$ & 0 & 0.219 \\
\hline Neck & $32(11.1 \%)$ & $12(9.0 \%)$ & 0.646 \\
\hline Elbow & $12(4.1 \%)$ & $4(3.0 \%)$ & 0.770 \\
\hline Hand & $22(7.5 \%)$ & $10(7.5 \%)$ & 1.000 \\
\hline Abdomen & $4(1.4 \%)$ & 0 & 0.412 \\
\hline
\end{tabular}

${ }^{\mathrm{a}}$ Each patient may have had more than one site of pain.

Table 4. Quality of Pain and Associated Symptoms Among the Chronic and Subacute Pain Groups

\begin{tabular}{|c|c|c|c|}
\hline & Chronic Pain, $n=292$ & Subacute Pain, $n=134$ & P Value \\
\hline Burning pain & $80(27.4 \%)$ & $26(19.4 \%)$ & 0.099 \\
\hline Pressure pain & $42(14.4 \%)$ & $20(14.9 \%)$ & 1.000 \\
\hline Sharp pain & $32(11.0 \%)$ & $10(7.5 \%)$ & 0.343 \\
\hline Dull pain & $36(12.3 \%)$ & $6(4.5 \%)$ & 0.019 \\
\hline Dagger pain & $12(4.1 \%)$ & $2(1.5 \%)$ & 0.265 \\
\hline Persistent pain & $58(19.9 \%)$ & $14(10.4 \%)$ & 0.023 \\
\hline Night pain & $24(8.2 \%)$ & $20(14.9 \%)$ & 0.048 \\
\hline Morning pain & $22(7.5 \%)$ & $18(13.4 \%)$ & 0.079 \\
\hline Increasing pain & $18(6.2 \%)$ & $4(3.0 \%)$ & 0.254 \\
\hline Intolerable pain & $30(10.3 \%)$ & $6(4.5 \%)$ & 0.070 \\
\hline Pain during sleep & $20(6.8 \%)$ & $10(7.5 \%)$ & 0.979 \\
\hline Anxiety & $104(35.6 \%)$ & $38(28.4 \%)$ & 0.336 \\
\hline Depression & $66(22.6 \%)$ & $22(16.4 \%)$ & 0.103 \\
\hline Fear & $4(1.4 \%)$ & $2(1.5 \%)$ & 1.000 \\
\hline Fatigue & $54(18.5 \%)$ & $26(19.4 \%)$ & 0.929 \\
\hline
\end{tabular}

${ }^{\mathrm{a}}$ Each patient may have had more than one form of pain or associated symptoms.

the United Kingdom were women. Chronic pain appears to have a direct relationship with age, and is more common among elderly people (13). In our study, the patients with chronic pain were older than those with subacute pain (57.2 \pm 15.3 vs. $53.5 \pm 15.9 \mathrm{y} ; \mathrm{P}=0.022$ ).

Chronic pain, especially lower back pain, is associated with increased levels of obesity $(19,20)$. Evidence indicates that the positive relationship between obesity and chronic pain is partially imposed by the over-loading of weight-bearing joints, including the spine, hip, and knee (21). Nonetheless, Brox et al. (22). found that sex, age, height, and body weight were not significantly different between the patients with subacute and chronic low back pain. In our study, body weight was not significantly differ- ent between the two groups, but the patients with chronic pain had a higher BMI than those with subacute pain $(\mathrm{P}=$ 0.010).

The most frequent site of pain in the patients who referred to the pain clinic in the present study was the lower back (62.4\%), followed by pain in the leg and foot (39.9\%), knee (24.4\%), and hip (18.8\%). In the study by Torrance et al. (2006) (3), the most common sites of pain were the back (69.9\%), leg and foot (64.6\%), neck and shoulder (62.6\%), and hip (42.2\%). Nevertheless, most of the patients complained of pain in more than one site. Boulanger et al. (2007)(23) in Canada reported a similar pattern in the body sites of non-cancer-related chronic pain, and cited that the most common sites were the lower back, leg, knee, neck, 
Table 5. Pain Severity Measured by the Numerical Rating Scale for the Chronic and Subacute Pain Groups

\begin{tabular}{lcc}
\hline & Chronic Pain, $\mathbf{n}=\mathbf{2 9 2}$ & Subacute Pain, $\mathbf{n}=\mathbf{1 3 4}$ \\
\hline Maximum severity reported & $8.89 \pm 1.47$ & $8.33 \pm 1.78$ \\
Minimum severity reported & $3.42 \pm 1.85$ & $3.18 \pm 1.86$ \\
Mean pain severity & $6.26 \pm 2.31$ & $5.67 \pm 2.33$ \\
\hline
\end{tabular}

head, and hip (in order).

In our study, the patients reported various qualities of pain, such as burning (24.9\%), persistence (16.9\%), and pressure $(14.6 \%)$ (Table 4$)$. Most pain qualities were similar between the chronic and subacute pain groups, except for dull and persistent pain, which was more common in the chronic pain group $(\mathrm{P}<0.05)$. Different pain qualities can affect the sensation of pain and the psychological aspects of pain perception. For example, Jensen et al. (24) reported that particular pain qualities including sharpness, sensitivity, and itchiness appear to play a substantial and exclusive role in the sensation of pain and global pain severity and unpleasantness.

The most common symptoms associated with pain were anxiety, depression, fatigue, and fear in the present study; there were no significant differences between the two study groups in this regard (Table 4). Some studies have found similar features $(25,26)$. For instance, Bair et al. (26) concluded that the coexistence of chronic pain with depression and anxiety was strongly related to more severe pain, increased disability, and poorer health-related quality of life. In their study, $54 \%$ of the patients reported pain only, 20\% complained of pain concomitant with depression, $3 \%$ had pain and anxiety, and $23 \%$ suffered from pain, depression, and anxiety.

In our study, mean pain severity was higher in the chronic pain group (numerical rating scale $=6.26 \pm 2.31$ ) than in the subacute pain group $(5.67 \pm 2.33)(\mathrm{P}=0.015)$. In the study by Torrance et al. (3), mean pain severity was 5 \pm 1.5 on the numerical rating scale. Bair et al. (26) showed the severity of $5.3 \pm 0.6$ in their chronic pain patients and reported that it increased with the presence of anxiety or depression.

\subsection{Conclusions}

Our study revealed that a notable percentage (31.5\%) of the patients referring to the pain clinic complained of experiencing pain in the preceding 12 weeks (subacute pain). These patients were somewhat different from those presenting with chronic pain vis-a-vis demographic and clinical features. About 59\% of the patients were women. The patients with chronic pain were older than those with subacute pain and had higher BMIs. Occupational status was also different between the two groups, with self- employment being less frequent among the chronic pain group. The patients with chronic pain had a higher prevalence of opium addiction. The most commonly reported site of pain for all patients was the lower back, followed by pain in the leg and foot, knee, hip, and neck. There were no differences in terms of the pain sites between the two groups, except for knee pain, which was more common in the chronic pain group. The patients with chronic pain had a higher frequency of obscure and persistent pain, while those with subacute pain experienced more night pain.

\section{Acknowledgments}

We especially thank the staff of Shahid Beheshti University of Medical Sciences and Akhtar hospital's pain clinic for their kind contributions to data collection from the patients referring to the pain clinic.

\section{Footnotes}

Authors' Contribution: Seyed Masoud Hashemi conducted the study and supervised the project. Ramin Rohanifar collected all data from patients and contributed to the research design planning and manuscript writing. Rasoul Azarfarin contributed to the manuscript writing and statistical analyses. Seyed Sajjad Razavi contributed to the research design planning and data collection. Sirous Momenzadeh contributed to the manuscript writing, data collection, and submission of the manuscript.

Funding/Support: There was no external funding provided for the preparation of this manuscript.

\section{References}

1. Ahangari A. Prevalence of chronic pelvic pain among women: an updated review. Pain Physician. 2014;17(2):141-7. [PubMed: 24658485].

2. Smith BH, Elliott AM, Chambers WA, Smith WC, Hannaford PC, Penny K. The impact of chronic pain in the community. Fam Pract. 2001;18(3):292-9. [PubMed: 11356737].

3. Torrance N, Smith BH, Bennett MI, Lee AJ. The epidemiology of chronic pain of predominantly neuropathic origin. Results from a general population survey. J Pain. 2006;7(4):281-9. doi: 10.1016/j.jpain.2005.11.008. [PubMed: 16618472]. 
4. Shmagel A, Foley R, Ibrahim H. Epidemiology of chronic low back pain in US adults: National Health and Nutrition Examination Survey 2009-2010. Arthritis Care Res (Hoboken). 2016 doi: 10.1002/acr.22890. [PubMed: 26991822].

5. Liang En W, Sin D, Wen Qi C, Zong Chen L, Shibli S, Choon-Huat Koh G. Chronic Pain in a Low Socioeconomic Status Population in Singapore: A Cross-Sectional Study. Pain Med. 2016:115. doi: 10.1093/pm/pnv115. [PubMed: 26893120].

6. Zarei S, Bigizadeh S, Pourahmadi M, Ghobadifar MA. Chronic Pain and Its Determinants: A Population-based Study in Southern Iran. Korean JPain. 2012;25(4):245-53. doi: 10.3344/kjp.2012.25.4.245. [PubMed: 23091685].

7. Asghari A, Julaeiha S, Godarsi M. Disability and depression in patients with chronic pain: pain or pain-related beliefs?. Arch Iran Med. 2008;11(3):263-9. doi: 08113/AIM.006. [PubMed:18426316].

8. Jackson T, Thomas S, Stabile V, Han X, Shotwell M, McQueen K. Prevalence of chronic pain in low-income and middle-income countries: a systematic review and meta-analysis. Lancet. 2015;385 Suppl 2:S10. doi:10.1016/S0140-6736(15)60805-4. [PubMed: 26313056].

9. Royuela A, Kovacs FM, Campillo C, Casamitjana M, Muriel A, Abraira V. Predicting outcomes of neuroreflexotherapy in patients with subacute or chronic neck or low back pain. Spine J. 2014;14(8):1588-600. doi: 10.1016/j.spinee.2013.09.039. [PubMed: 24345468].

10. Markowski A, Sanford S, Pikowski J, Fauvell D, Cimino D, Caplan S. A pilot study analyzing the effects of Chinese cupping as an adjunct treatment for patients with subacute low back pain on relieving pain, improving range of motion, and improving function. J Altern Complement Med. 2014;20(2):113-7. doi: 10.1089/acm.2012.0769. [PubMed: 24299469].

11. Goertz M, Thorson D, Bonsell J, Bonte B, Campbell R, Haake B, et al. Adult acute and subacute low back pain. Institute for Clinical Systems Improvement [Internet]. Updated November. 2012:10-2.

12. van Hecke O, Torrance N, Smith BH. Chronic pain epidemiology and its clinical relevance. Br J Anaesth. 2013;111(1):13-8. doi: 10.1093/bja/aet123. [PubMed: 23794640].

13. Goldberg DS, McGee SJ. Pain as a global public health priority. BMC Public Health. 2011;11:770. doi: 10.1186/1471-2458-11-770. [PubMed: 21978149].

14. Imani F, Safari S. "Pain Relief is an Essential Human Right", We Should be Concerned about It. Anesth Pain Med. 2011;1(2):55-7. doi: 10.5812/kowsar.22287523.2306. [PubMed: 25729655].

15. Kamalipour H, Vafaei A, Parviz Kazemi A, Khademi S. Comparing the prevalence of chronic pain after sternotomy in patients undergoing coronary artery bypass grafting using the internal mammary artery and other open heart surgeries. Anesth Pain Med. 2014;4(3):17969. doi: 10.5812/aapm.17969. [PubMed: 25289372].

16. Hill RJ, Chopra P, Richardi T. Rethinking the psychogenic model of complex regional pain syndrome: somatoform disorders and complex regional pain syndrome. Anesth Pain Med. 2012;2(2):54-9. doi 10.5812/aapm.7282. [PubMed: 24223338].

17. Kamali F, Panahi F, Ebrahimi S, Abbasi L. Comparison between massage and routine physical therapy in women with sub acute and chronic nonspeci fi c low back pain. J Back Musculoskelet Rehabil. 2014;27(4):475-80. doi: 10.3233/BMR-140468. [PubMed: 24867893].

18. Kent $P$, Laird R, Haines $T$. The effect of changing movement and posture using motion-sensor biofeedback, versus guidelines-based care, on the clinical outcomes of people with sub-acute or chronic low back pain-a multicentre, cluster-randomised, placebo-controlled, pilot trial. BMC Musculoskelet Disord. 2015;16:131. doi: 10.1186/s12891-0150591-5. [PubMed: 26022102].

19. Chou L, Brady SR, Urquhart DM, Teichtahl AJ, Cicuttini FM, Pasco JA, et al. The Association Between Obesity and Low Back Pain and Disability Is Affected by Mood Disorders: A Population-Based, CrossSectional Study of Men. Medicine (Baltimore). 2016;95(15):3367. doi: 10.1097/MD.0000000000003367. [PubMed: 27082599].

20. Shemory ST, Pfefferle KJ, Gradisar IM. Modifiable Risk Factors in Patients With Low Back Pain. Orthopedics. 2016;39(3):413-6. doi: 10.3928/01477447-20160404-02. [PubMed: 27064774].

21. Paley C, Johnson MI. Chronic pain in the obese population: is exercise the key?.Pain Manag. 2016;6(2):121-3. doi:10.2217/pmt.15.64. [PubMed: 26912195].

22. Brox JI, Storheim K, Holm I, Friis A, Reikeras O. Disability, pain, psychological factors and physical performance in healthy controls, patients with sub-acute and chronic low back pain: a case-control study. J Rehabil Med. 2005;37(2):95-9. doi:10.1080/16501970410017738. [PubMed 15788344].

23. Boulanger A, Clark AJ, Squire P, Cui E, Horbay GL. Chronic pain in Canada: have we improved our management of chronic noncancer pain?. Pain Res Manag. 2007;12(1):39-47. [PubMed:17372633].

24. Jensen MP, Dworkin RH, Gammaitoni AR, Olaleye DO, Oleka N, Galer BS. Do pain qualities and spatial characteristics make independent contributions to interference with physical and emotional functioning?. J Pain. 2006;7(9):644-53. doi: 10.1016/j.jpain.2006.02.012. [PubMed: 16942950].

25. Alaei F, Davari PN, Alaei M, Azarfarin R, Soleymani E. Postoperative outcome for hyperglycemic pediatric cardiac surgery patients. Pediatr Cardiol. 2012;33(1):21-6. doi: 10.1007/s00246-011-0060-3. [PubMed: 21850482].

26. Bair MJ, Wu J, Damush TM, Sutherland JM, Kroenke K. Association of depression and anxiety alone and in combination with chronic musculoskeletal pain in primary care patients. Psychosom Med. 2008;70(8):890-7. doi: 10.1097/PSY.0b013e318185c510. [PubMed: 18799425]. 